\title{
The Khuzestan Comprehensive Health Study (KCHS): Methodology and Profile of Participants
}

\author{
Bahman Cheraghian, PhD $^{1 *}$; Maryam Sharafkhah, PhD ${ }^{2,3 *}$; Zahra Mohammadi, PhD ${ }^{2 *}$; Sanam Hariri, MD²; Zahra Rahimi, MSc ${ }^{4}$; Leila \\ Danehchin, MD ${ }^{5}$; Yousef Paridar, MD ${ }^{6}$; Farhad Abolnejadian, MD,8; Mohammad Noori, MD'; Seyed Ali Mard, PhD ${ }^{10}$; Sareh Eghtesad, PhD²; \\ Amaneh Shayanrad, MSc'2; Arash Etemadi, PhD ${ }^{11}$; Reza Ghanbari, PhD²; Farnaz Hashemi, PhD²; Sahar Masoudi, MSc²; Ali Akbar Shayesteh, \\ $\mathrm{MD}^{12^{*}}$; Hossein Poustchi, MD, PhD ${ }^{2 *}$
}

${ }^{1}$ Alimentary Tract Research Center, Ahvaz Jundishapur University of Medical Sciences, Ahvaz, Iran

${ }^{2}$ Liver and Pancreatobiliary Diseases Research Center, Digestive Diseases Research Institute, Tehran University of Medical Sciences, Tehran, Iran

${ }^{3}$ Department of Bio-statistics and Epidemiology, Faculty of Public Health, Tehran University of Medical Sciences, Tehran, Iran ${ }^{4}$ Department of Biostatistics and Epidemiology, School of Public Health, Ahvaz Jundishapur University of Medical Sciences, Ahvaz, Iran ${ }^{5}$ Faculty of Medicine, Behbahan University of Medical Sciences, Behbahan, Iran

${ }^{6}$ Faculty of Medicine, Dezful University of Medical Sciences, Dezful, Iran

'Department of Clinical Immunology and Allergy, Ahvaz Jundishapur University of Medical Sciences, Ahvaz, Iran ${ }^{8}$ Shoushtar School of Medical Sciences and Health Services, Shoushtar, Iran

${ }^{9}$ Abadan Faculty of Medical Sciences, Abadan, Iran

${ }^{10}$ Physiology Research Center, School of Public Health, Ahvaz Jundishapur University of Medical Sciences, Ahvaz, Iran "Division of Cancer Epidemiology and Genetics, National Cancer Institute, Bethesda, Maryland, United States

${ }^{12}$ Alimentary Tract Research Center, School of Medicine, Ahvaz Jundishapur University of Medical Sciences, Ahvaz, Iran

\begin{abstract}
Background: Non-communicable diseases (NCDs) are the leading cause of death worldwide, with a disproportionally rising burden among low- and middle-income populations. While preventable risk factors highly contribute to this burden, populationbased studies assessing these factors and the health status of these populations, are scarce.

Methods: The Khuzestan Comprehensive Health Study (KCHS) - a cross sectional study —was conducted between 2016-2019, including 30,506 Iranians aged 20 to 65 years, from 27 counties of Khuzestan province, southwest of Iran. KCHS aimed to provide a comprehensive health overview by investigating the prevalence and risk factors of NCDs and psychological disorders, along with viral hepatitis as a common communicable disease. Upon registration, $15 \mathrm{~mL}$ of blood and anthropometric measurements were obtained from participants. Afterwards, several interviewer-administered questionnaires were completed to gather data on demographics, socioeconomic status, sleep quality, physical activity, lifestyle habits, nutrition, and medical history.

Results: The mean \pm SD age of participants was $41.7 \pm 11.9$ years. The majority were female $(64.3 \%)$, of the Arab ethnicity $(49 \%)$, married $(83 \%)$, and urban residents $(73.1 \%)$. About $70 \%$ had an educational level below high school diploma. Overall, 10.8\%, $5.2 \%$, and $2.8 \%$ of participants had used cigarettes, hookah, and drugs at least once in their lifetime, respectively. While body mass index and serum cholesterol levels were higher in females, blood pressure was higher in males $(P<0.001)$.

Conclusion: KCHS assessed many aspects of health in the Khuzestan province. In addition to develop a biobank along with a comprehensive dataset, KCHS will serve as a valuable infrastructure for future research.

Keywords: Epidemiology, Iran, Khuzestan, Non-communicable diseases, Risk factors

Cite this article as: Cheraghian B, Sharafkhah M, Mohammadi Z, Hariri S, Rahimi Z, Danehchin L, et al. The Khuzestan Comprehensive Health Study (KCHS): methodology and profile of participants. Arch Iran Med. 2020;23(10):653-657. doi: 10.34172/aim.2020.82.
\end{abstract}

Received: September 9, 2020, Accepted: September 16, 2020, ePublished: October 1, 2020

\section{Introduction}

Over two-thirds of deaths worldwide are attributable to non-communicable diseases $\quad(\mathrm{NCDs}),{ }^{1}$ rising disproportionately among marginalized as well as low- and middle-income populations. ${ }^{2}$ The rising burden of NCDs is mainly driven by rapid urbanization and increased life expectancy. Still, preventable risk factors mainly contribute to this burden and present targets for control measures. ${ }^{3}$ Despite recent lifestyle changes, limited population-based studies are available to assess the health status of people in low- and middle-income regions, including the Middle East. ${ }^{4}$

Fifteen million premature deaths occur annually due to NCDs, over $85 \%$ of which occur in low- and middleincome countries. ${ }^{5} \mathrm{NCD}$ deaths are mainly caused by cardiovascular diseases, neoplasms, chronic respiratory diseases, and diabetes, imposing detrimental economic and health burdens on communities. ${ }^{1,2}$ In addition to 
traditional risk factors, such as smoking, dietary and lifestyle habits, nontraditional factors like pollution and climate change have emerged in recent decades, predisposing many countries to numerous health hazards. ${ }^{3}$

Southwestern Iran is a unique region, given its natural conditions and geological, climatic, and soil characteristics. ${ }^{6}$ Recently, it has been exposed to dust storms, extremely high temperatures, air pollution, and soil contamination, all of which could threaten the health of its residents. ${ }^{7,8}$ Furthermore, the southern border of Iran, mainly Khuzestan province was heavily affected by the Iran-Iraq war (1980-1988). Many cities were partially or completely destructed and much of the infrastructure in the area was destroyed during that time, also debilitating the physical and psychosocial health of its residents.

Despite the above-mentioned facts, no cross-sectional study has yet assessed the health status of the people of Khuzestan province. Also, given the impact of war on mental health, a psychological assessment is particularly important for those residing in the region, but has not yet been completed. ${ }^{\text {' }}$ Therefore, the Khuzestan Comprehensive Health Study (KCHS) was conducted with the aim to provide a comprehensive overview of health in Khuzestan, including the prevalence and risk assessment of non-communicable and psychological diseases, as well as viral hepatitis as a common communicable disease in Iran. In this manuscript, the methodology used in KCHS is described in detail and the profile of the participants is presented.

\section{Materials and Methods}

Study Design

KCHS was a cross-sectional study conducted between October 2016 and November 2019 in 30,500 Iranian adults from 27 counties of Khuzestan province. This large population-based study was funded by the National Institute for Medical Research Development (NIMAD) and the Iranian Blood Transfusion Organization (IBTO), and executed by the Digestive Diseases Research Institute (DDRI), Jundishapur and Dezful Universities of Medical Sciences, as well as Abadan and Behbahan Faculties of Medicine. The KCHS protocol was approved by the ethics committee of NIMAD (IR.NIMAD.REC.1394.002).

\section{Study Site}

The Khuzestan province is located in southwestern Iran, sharing a border with Iraq to the west and lying north of the Persian Gulf (Figure 1). The province covers an area of about $64000 \mathrm{~km}^{2}$ and is home to a population of nearly 4936000 individuals of different ethnicities, including Arabs, Lurs, and Fars. Khuzestan has one of the hottest climates in Iran with an average daily high temperature of $35^{\circ} \mathrm{C}$, and reaching as high as $49^{\circ} \mathrm{C}$ in the summers. This province is notable for its oil resources and is a central oilproducing region. ${ }^{10}$

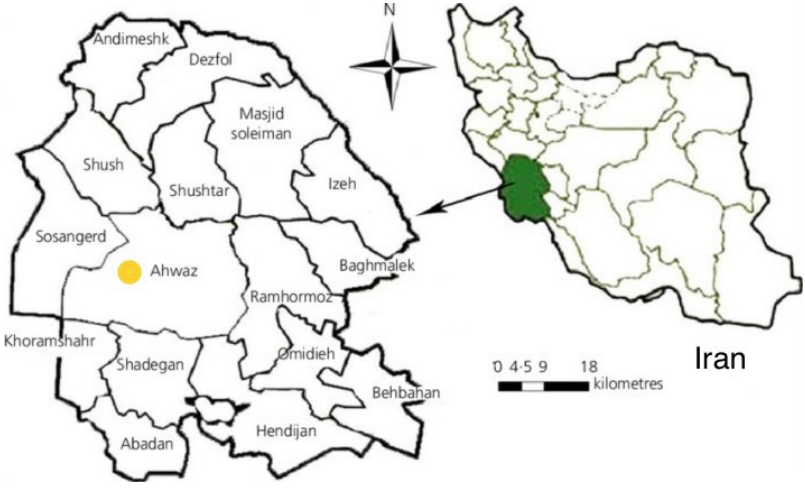

Figure 1. Map of Khuzestan Province and its Counties in Iran.

\section{Study Population}

The study population included all men and women in the age range of 20 to 65 years, residing in urban and rural areas of the Khuzestan, for a minimum duration of 12 months prior to the study recruitment. Exclusion criteria were unwillingness to participate and any mental, psychological, or physical disabilities that inhibit the completion of questionnaires or other steps.

\section{Sample Size}

The sample size required to estimate the prevalence of any health conditions in the province with $1 \%$ margin of error was calculated. A $50 \%$ baseline prevalence to maximize the required sample size was assumed. As such, a sample size of 30,000 will provide a power greater than $90 \%$ for estimations, and was chosen for this study (Figure 2).

\section{Sampling Method}

Primary care centers, called "Health Houses," have long been established throughout Iran. Each Health House covers a target population of about 1500 individuals, archiving the population census and their health records. ${ }^{11}$ The population census at the Health Houses were used as the sampling frame, with the aim to recruit 30 random individuals from each Health House. To obtain a geographically representative sample, the population of all 27 counties in the province were stratified and a random sample of Health Houses in each county were selected. The number of Health Houses included from each county was determined based on the proportion of the total province population living in that county. Consequently, 1,079 random Health Houses (780 urban and 299 rural), were selected across the province, and thereafter, 30 individuals who fulfilled the eligibility criteria were randomly selected from the population covered by each Health House.

\section{Data Collection \\ I. Invitation}

Based on the above-mentioned sampling methods, trained staff performed face-to-face recruitment of potential participants, explained the aims of the study and if they 


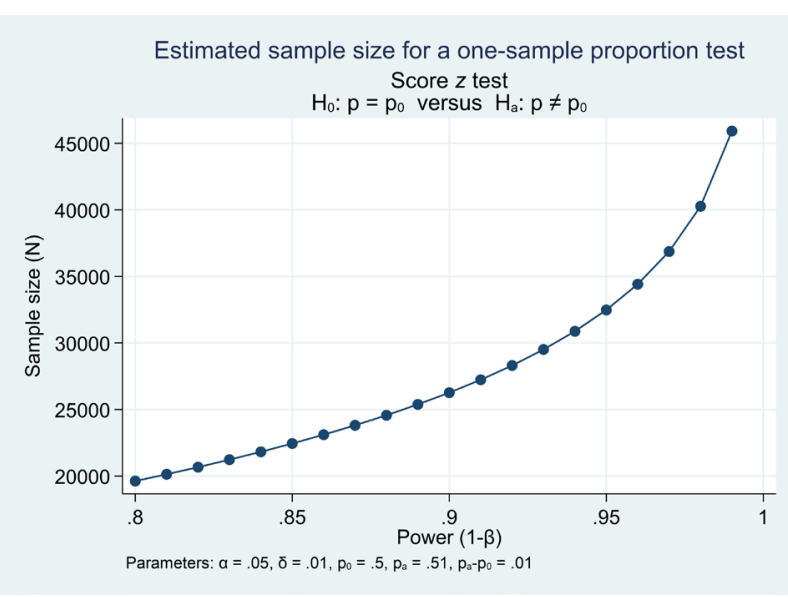

Figure 2. The Estimated Sample Size for a One-Sample Proportion Test.

accepted to participate, they were instructed to visit a predetermined study site. The study sites were health centers, Health Houses, schools and mosques, located in close proximity of the participants' homes.

\section{Registration}

Upon arrival at the study site, written informed consent was obtained from each participant, who was then registered using credible personal identification documents. A unique 11-digit code was assigned to each individual; all questionnaires and biological samples were labeled by these codes.

\section{Biological Sampling, Laboratory Assessment and Biobanking}

After registration, $15 \mathrm{~mL}$ blood, including one clot tube $(6 \mathrm{~mL})$ and one EDTA tube $(9 \mathrm{~mL})$ (KANG JIAN, China), was obtained from participants by trained staff, using Vacutainers (Greiner Bio-One International GmbH, Kremsmunster, Austria). All participants were fasted for 8 to 12 hours at the time of sampling. Blood was transferred to the reference lab within three hours of sampling, stored in Cool boxes (Pole Ideal Pars, Iran), at $4^{\circ} \mathrm{C}$.

Blood samples were centrifuged at $3000 \mathrm{rpm}$ (Sigma, Germany) for 10 minutes to separate the serum. Later, serum levels of fasting blood sugar (FBS), urea, creatinine (Cr), triglycerides (TG), cholesterol (Chol), high-density lipoproteins (HDL), alkaline phosphatase (ALP), alanine aminotransferase (ALT), aspartate aminotransferase (AST) and gamma-glutamyl transferase (GGT) were measured by BT 1500 AutoAnalyzer (Biotecnica Instruments, Italy), using commercial kits (Pars Azmun, Iran). A complete blood count (CBC) was performed by hematology AutoAnalyzer (Nihon Kohden 6510-k, Japan). Also, a serum sample $(500 \mu \mathrm{L})$ was transferred at $-20^{\circ} \mathrm{C}$ to IBTO in Tehran for serologic evaluation of hepatitis $B$ and $\mathrm{C}$ viruses. Blood chemistry and serology results were returned to participants. In case of any abnormal findings, participants were referred to family physicians or specialists for further evaluation.

In addition to the biochemical and hematological tests, fractioned samples of whole blood (1 tube), plasma ( 3 tubes), buffy coat (1 tube) and serum (1 tube) were biobanked for each individual in sterile barcoded cryotubes (Micronic, Netherlands) at $-80^{\circ} \mathrm{C}$.

\section{Anthropometric Measurements}

Given the lower occurrence of measurement errors in the morning, anthropometric measurements were made after blood collection while participants were still fasted. Weight $(\mathrm{kg})$ and height $(\mathrm{cm})$ were measured by the Seca 762 Mechanical Flat Scale and Seca 206 Bodymeter Measuring Tape with Wall Stop, respectively. The Seca 203 Circumference Measuring Tape was used for waist, hip, and wrist circumferences $(\mathrm{cm})$. All measurements were made following National Institutes of Health protocols. ${ }^{12}$ Breakfast was provided prior to the completion of the following steps.

\section{Questionnaires}

The KCHS questionnaire set consisted of five major sections: general, medical, nutrition, gastrointestinal, and psychological. While the general and medical sections were completed for all participants, other sections were only completed for a subsample. Questionnaires were completed by trained interviewers. An explanation of each section is as follows.

\section{$V-A$. General Section}

The general questionnaire included information on demographic characteristics, socioeconomic status, sleep quality, and physical activity (International Physical Activity Questionnaires [IPAQ]). ${ }^{13}$

\section{V-B. Medical Section}

This section contained questions regarding the personal and family history of chronic diseases, medication use, lifestyle and personal habits, and risk factors of blood-borne disease transmission. In addition, a fertility questionnaire unique to each gender was administered to all participants. As part of the medical evaluation, blood pressure was measured twice in each arm, in the sitting position, using aneroid sphygmomanometers (Riester, Germany).

\section{V-C. Nutrition Section}

Ten percent of participants were randomly selected to complete an 86-item qualitative food frequency questionnaire (FFQ), with 9 response categories used to indicate their usual food consumption frequency in the year prior to recruitment. Among the selected participants, 2,831 (9.3\% of total) individuals completed this section.

\section{V-D. Gastrointestinal Section}

Ten percent of participants were randomly selected 
to complete the irritable bowel syndrome (IBS) and gastroesophageal reflux disease (GERD) questionnaires, containing 21 and 10 questions, respectively. Overall, $1,853(6.1 \%)$ participants completed this section.

\section{V-E. Psychological Section}

To complete the psychological section, $25 \%$ of participants were randomly selected, of whom, 6,935 (22.7\%) individuals completed the following four questionnaires: Depression Anxiety Stress Scales (DASS), Life Events Checklist (LEC), Post-traumatic Stress Disorder Checklist (PCL), and War Exposure.

\section{Quality Assurance and Quality Control}

The main tool used to control the quality of data collected, was a smart data server that minimized common errors in data entry, including missing and out-of-range values. To ensure consistent data collection, the same equipment and devices were used at all study sites and executing teams were trained by the same instructors. Also, to ensure the compliance of study procedures with the KCHS protocol, the executive teams were frequently evaluated through unexpected visits.

Also, to ensure the compliance of study procedures with the KCHS protocol, the executive teams were frequently evaluated through unexpected visits.

\section{Results}

A total of 30,506 participants were enrolled in the study, of whom 19,604 (64.3\%) were female. The mean \pm SD age of participants was $41.7 \pm 11.9$ years. Demographic characteristics of the participants are presented in Table 1. The majority of participants were of the Arab ethnicity $(49.0 \%)$, were married $(83.0 \%)$, and resided in urban areas $(73.1 \%)$.

Health-related characteristics of study participants are described in Table 2. Overall, $10.8 \%$ and $5.2 \%$ of participants had smoked cigarettes and hookah in their lifetime, respectively; $2.1 \%$ had consumed alcohol, and $2.8 \%$ had used drugs. The prevalence of cigarette and hookah use were higher in males, compared to females (22 and 5-folds, respectively), and the prevalence of alcohol and drug use was much less among females $(P<0.001)$. Among all participants, $42.9 \%$ had moderate, and 31.1\% had low physical activity levels; the remaining 26\% were classified as high. While the prevalence of high physical activity was similar in both genders, the moderate level was more prevalent among females, compared to males $(P<0.001)$ (Table 2).

The mean \pm SD of body mass index $(\mathrm{BMI})$ was $27.63 \pm$ 5.3 , and higher in females $(P<0.001)$. For total cholesterol, the mean \pm SD was $193.1 \pm 47.5$; which was higher in females $(P<0.001)$. In contrast, the triglyceride levels were relatively higher in males $(P<0.001)$. Also, mean systolic and diastolic blood pressures were higher among male
Table 1. Demographic Characteristics of KCHS Participants

\begin{tabular}{|c|c|c|}
\hline Characteristics & & Total, No. (\%) \\
\hline \multirow{2}{*}{ Gender } & Male & $10902(35.7)$ \\
\hline & Female & $19604(64.3)$ \\
\hline \multirow{5}{*}{ Ethnicity } & Arab & $14929(49.0)$ \\
\hline & Bakhtiari & $6738(22.1)$ \\
\hline & Fars & $5621(18.5)$ \\
\hline & Lur & $2928(9.7)$ \\
\hline & Other & $226(0.7)$ \\
\hline \multirow{2}{*}{$\begin{array}{l}\text { Place of } \\
\text { residence }\end{array}$} & Urban & $22292(73.1)$ \\
\hline & Rural & $8214(26.9)$ \\
\hline \multirow{5}{*}{$\begin{array}{l}\text { Age group } \\
\text { (years) }\end{array}$} & $20-29$ & $5645(18.5)$ \\
\hline & $30-39$ & $8381(27.5)$ \\
\hline & $40-49$ & $7579(24.9)$ \\
\hline & $50-59$ & $6287(20.7)$ \\
\hline & $60-65$ & $2557(8.4)$ \\
\hline \multirow{3}{*}{ Marital status } & Married & $25325(83.0)$ \\
\hline & Single & $3644(12.0)$ \\
\hline & Divorced/widowed & $1537(5.0)$ \\
\hline \multirow{5}{*}{$\begin{array}{l}\text { Educational } \\
\text { level }\end{array}$} & None (Illiterate) & $6607(21.7)$ \\
\hline & Elementary school & $8237(27.0)$ \\
\hline & Middle school & $5246(17.2)$ \\
\hline & High school diploma & $6693(21.9)$ \\
\hline & University degree & $3718(12.2)$ \\
\hline
\end{tabular}

participants $(P<0.001)$.

\section{Discussion}

This is the first cross-sectional study assessing the prevalence and associated risk factors of NCDs, psychological disorders, and hepatitis $\mathrm{B}$ and $\mathrm{C}$ infections in the Khuzestan province of Iran. The findings of this study can help identify and better understand the health obstacles in the region and emerging health threats, in order to improve pertinent health policies. The main limitation of this study was the higher response rate in females in comparison to males. However, this limitation can be adjusted for in data analysis by reporting gender-specific or gender-standardized estimates. Given the importance of biobanking in the future of medicine, the samples stored in KCHS will serve as a valuable foundation for future research, especially in the identification of disease biomarkers and genetic variations of NCDs.

\section{Authors' Contribution}

$\mathrm{HP}, \mathrm{AAS}, \mathrm{BC}, \mathrm{ZM}$ and $\mathrm{SE}$ contributed to the design, study execution and manuscript writing. AS, ZR, LD, YP, FA, MN, AM, SM and FH contributed to data collection and manuscript writing. MS, AE, RG and $\mathrm{SH}$ contributed to data analysis and manuscript writing.

\section{Conflict of Interest Disclosures}

Authors have no conflicts to declare. 
Table 2. Health-related Characteristics of KCHS Participants

\begin{tabular}{|c|c|c|c|c|}
\hline Characteristics & Male & Female & Total & $P$ value \\
\hline Cigarette smoking, ever ${ }^{\mathrm{a}}$ & $3043(28.0)$ & $255(1.3)$ & $3298(10.8)$ & $<0.001$ \\
\hline Hookah smoking, ever ${ }^{\mathrm{a}}$ & $1158(10.6)$ & $435(2.2)$ & $1593(5.2)$ & $<0.001$ \\
\hline Alcohol use, ever ${ }^{\mathrm{a}}$ & $617(5.7)$ & $31(0.2)$ & $648(2.1)$ & $<0.001$ \\
\hline Drug use, ever ${ }^{\mathrm{a}}$ & $809(7.4)$ & $42(0.2)$ & $851(2.8)$ & $<0.001$ \\
\hline \multicolumn{5}{|l|}{ Physical activity } \\
\hline Low & $4686(43.1)$ & $4763(24.3)$ & $9615(31.1)$ & \multirow{3}{*}{$<0.001$} \\
\hline Moderate & $3445(31.7)$ & $9615(49.2)$ & $13060(42.9)$ & \\
\hline High & $2732(25.2)$ & $5182(26.5)$ & $7914(26.0)$ & \\
\hline Body mass index $(\mathrm{BMI})^{\mathrm{b}}$ & $26.50 \pm 4.7$ & $28.25 \pm 5.5$ & $27.63 \pm 5.3$ & $<0.001$ \\
\hline Systolic blood pressure $(\mathrm{mm} \mathrm{Hg})^{\mathrm{b}}$ & $118.34 \pm 14.8$ & $114.42 \pm 15.0$ & $115.82 \pm 15.1$ & $<0.001$ \\
\hline Diastolic blood pressure $(\mathrm{mm} \mathrm{Hg})^{\mathrm{b}}$ & $76.48 \pm 10.6$ & $73.13 \pm 11.2$ & $74.33 \pm 11.1$ & $<0.001$ \\
\hline Fasting blood sugar & $110.99 \pm 48.0$ & $110.33 \pm 47.5$ & $110.57 \pm 47.6$ & 0.247 \\
\hline Total cholesterol ${ }^{\mathrm{b}}$ & $191.45 \pm 48.6$ & $194.05 \pm 46.8$ & $193.12 \pm 47.5$ & $<0.001$ \\
\hline High-density lipoproteins ${ }^{b}$ & $46.43 \pm 10.4$ & $53.46 \pm 11.8$ & $50.95 \pm 11.8$ & $<0.001$ \\
\hline Triglycerides ${ }^{\mathrm{b}}$ & $169.94 \pm 117.73$ & $141.01 \pm 90.6$ & $151.36 \pm 102.1$ & $<0.001$ \\
\hline
\end{tabular}

${ }^{\text {a }}$ Data are presented as number and percentage.

${ }^{\mathrm{b}}$ Data are presented as mean \pm standard deviation (SD).

\section{Ethical Statement}

The study protocol was approved by the ethics committee of the National Institute for Medical Research Development (IR.NIMAD. REC.1394.002).

\section{Funding}

This project was funded by the National Institute for Medical Research Development (NIMAD, Grant number: 940406).

\section{Acknowledgements}

We want to thank the participating universities and faculties for their cooperation, our study sites for their appreciable efforts, and the people of Khuzestan for their kind participation in this study.

\section{References}

1. Naghavi M, Abajobir AA, Abbafati C, Abbas KM, Abd-Allah F, Abera SF, et al. Global, regional, and national age-sex specific mortality for 264 causes of death, 1980-2016: a systematic analysis for the Global Burden of Disease Study 2016. Lancet. Lancet. 2017;390(10100):1151-210. doi: 10.1016/S01406736(17)32152-9.

2. WHO. Global Health Observatory (GHO) data. NCD mortality and morbidity. 2016. Available from: https://www. who.int/gho/ncd/mortality_morbidity/en/.

3. Benziger CP, Roth GA, Moran AE. The Global Burden of Disease Study and the Preventable Burden of NCD. Glob Heart. 2016;11(4):393-397. doi: 10.1016/j.gheart.2016.10.024.

4. Williams J, Allen L, Wickramasinghe K, Mikkelsen B, Roberts $\mathrm{N}$, Townsend N. A systematic review of associations between non-communicable diseases and socioeconomic status within low- and lower-middle-income countries. J Glob Health. 2018;8(2):020409. doi: 10.7189/jogh.08.020409.

5. WHO. Noncommunicable diseases. 2018. Available from: https://www.who.int/news-room/fact-sheets/detail/ noncommunicable-diseases.

6. Moghaddam A. Southwestern Iran. In: Potts DT, ed. A companion to the archaeology of the ancient Near East. John Wiley \& Sons; 2012:512-30. doi: 10.1002/9781444360790.

7. Dastoorpoor M, Goudarzi G, Khanjani N, Idani E, Aghababaeian $H$, Bahrampour A. Lag time structure of cardiovascular deaths attributed to ambient air pollutants in Ahvaz, Iran, 2008-2015. Int J Occup Med Environ Health. 2018;31(4):459-473. doi: 10.13075/ijomeh.1896.01104.

8. Ghanavati N, Nazarpour A, De Vivo B. Ecological and human health risk assessment of toxic metals in street dusts and surface soils in Ahvaz, Iran. Environ Geochem Health. 2019;41(2):875-891. doi: 10.1007/s10653-018-0184-y.

9. Jamshidi F, Nazari I, Malayeri HT, Rahimi Z, Cheraghi M. Pattern of drug abuse in addicts self-referred drug rehabilitation centers in Khuzestan province - Iran, 2014-2015. Arch Med Sadowej Kryminol. 2016;66(1):1-12. doi: 10.5114/ amsik.2016.62330.

10. Britannica. Khūzestān, geographical region, Iran. Available from: geographical region, Iran.

11. Mehrdad R. Health system in Iran. JMAJ. 2009;52(1):69-73.

12. Centers for Disease Control and Prevention. National Center for Health Statistics. National Health and Nutrition Examination Survey. Available from: https://www.cdc.gov/ nchs/nhanes/index.htm.

13. Moghaddam MB, Aghdam FB, Jafarabadi MA, Allahverdipour $\mathrm{H}$, Nikookheslat SD, Safarpour S. The Iranian Version of International Physical Activity Questionnaire (IPAQ) in Iran: content and construct validity, factor structure, internal consistency and stability. World Appl Sci J. 2012;18(8):107380. doi: 10.5829/idosi.wasj.2012.18.08.754. 